\title{
Investigation of AlInN barrier ISFET structures with GaN capping for pH detection
}

\author{
T. Brazzini , A. Bengoechea-Encabo, M.A. Sánchez-García, F. Calle
}

\begin{abstract}
A B S T R A C T
The $\mathrm{pH}$ response of GaN/AlInN/AlN/GaN ion-sensitive field effect transistor (ISFET) on Si substrates has been characterized. We analyzed the variation of the surface potential $\left(\Delta V_{\mathrm{sp}} / \Delta \mathrm{pH}\right)$ and current $\left(\Delta I_{\mathrm{ds}} / \Delta \mathrm{pH}\right)$ with solution $\mathrm{pH}$ in devices with the same indium content (17\%, in-plane lattice-matched to $\mathrm{GaN}$ ) and different AlInN thickness ( $6 \mathrm{~nm}$ and $10 \mathrm{~nm}$ ), and compared with the literature. The shrinkage of the barrier, that has the effect to increase the transconductance of the device, makes the 2-dimensional electron density (2DEG) at the interface very sensitive to changes in the surface.

Although the surface potential sensitivity to $\mathrm{pH}$ is similar in the two devices, the current change with $\mathrm{pH}\left(\Delta I_{\mathrm{ds}} / \Delta \mathrm{pH}\right)$, when biasing the ISFET by a $\mathrm{Ag} / \mathrm{AgCl}$ reference electrode, is almost $50 \%$ higher in the device with $6 \mathrm{~nm}$ AlInN barrier, compared to the device with $10 \mathrm{~nm}$ barrier. When measuring the current response ( $\Delta I_{\mathrm{ds}} / \Delta \mathrm{pH}$ ) without reference electrode, the device with thinner AllnN layer has a larger response than the thicker one, of a factor of $140 \%$, and that current response without reference electrode is only $22 \%$ lower than its maximum response obtained using reference electrode.
\end{abstract}

\section{Introduction}

After the first introduction of the concept of ion sensitive field effect transistor (ISFET) by Bergveld [1] the interest of solid-state based devices as chemical sensors has increased. Gate voltages induced by ions adsorbed onto the gate region of a semiconductor field effect transistor (FET) modulate the source-drain currents, realizing the concept of ISFET. The first device was fabricated on silicon with $\mathrm{SiO}_{2}$ as dielectric material on top of the gate region inducing a shift in the surface potential proportional to the ion concentration in the solution. Since then, the development has focused mainly on material selection to improve the performance of the ISFET as a pH sensor and on achieving integration between the ISFET and CMOS processing $[2,3]$.

In the last decade the interest in nitride-based sensors has increased both in the field of gas and ion-detection [4-11], especially since the first evidence of pH-sensitivity of GaN surfaces [6] and its application in $\mathrm{pH}-$ sensitive $\mathrm{AlGaN} / \mathrm{GaN}$ field effect transistors [7]. In particular, the $\mathrm{Ga}_{x} \mathrm{O}_{y}$ on the surface (native or promoted by wet oxidation) seems to follow the site-binding model [12] that explains the $\mathrm{pH}$ sensitivity. Compared to standard dielectric materials such as $\mathrm{SiO}_{2}, \mathrm{Si}_{3} \mathrm{~N}_{4}$ and $\mathrm{Al}_{2} \mathrm{O}_{3}$ used in commercial ISFET, improved sensitive response and robust surface properties can be ascribed to nitride materials like GaN [7] and InN [8]. Especially for chemical and biomedical applications in harsh environments, nitride robustness can be a solution to avoid or reduce possible damages and contaminations. Devices with high electron mobility transistor (HEMT) hetero-structures have been frequently used in $\mathrm{pH}$ detection mode [7,9]. In structures such as AlGaN/GaN $[9,10]$ or AlN/GaN [11], the conductivity of two dimensional electron gas (2DEG) located at the interface between the barrier and the $\mathrm{GaN}$ is affected by surface potential modifications.

Recently, the AlInN barrier HEMT is substituting the conventional AlGaN technology basically due to several facts. First of all, InAlN can be grown lattice matched (LM) to GaN for an indium composition of $16-18 \%$, reducing the strain present in a typical AlGaN-based transistor, and thus improving the long-term reliability of the device.

As InAlN can offer a high concentration of 2DEG density due to its much higher spontaneous polarization compared to AlGaN, a thinner barrier can be used. From a point of view of an open gate sensor, this provides an advantage regarding the sensitivity of the 2DEG to changes in the environment. A constant sheet charge density down to approximately $10 \mathrm{~nm}$ barrier thickness has been observed. Even at $5 \mathrm{~nm}$ a sheet charge density of around $1.6 \times 10^{13} \mathrm{~cm}^{-2}$ has been measured [13].

Due to the lack of lattice-matched materials, sapphire and silicon carbide are commonly used as substrates. Recently, the Si(111) substrate has attracted more attention because of its low cost, good thermal conductivity, availability of wafers up to $30 \mathrm{~cm}$ in diameter, and more especially, the possibility of integrating Si electronics on the same chip. Up to now, encouraging results have been achieved in the fabrication of devices based on $\mathrm{GaN} / \mathrm{Si}(1111)$ layers. InAIN barrier HEMTs grown on silicon substrate have been 
reported with channel electron sheet density of $2.6 \times 10^{13} \mathrm{~cm}^{-2}$ mobility $\mu=400 \mathrm{~cm}^{2} / \mathrm{Vs}$ and frequency operation up to $102 \mathrm{GHz}$ [14]. Therefore, it opens a way to a technology compatible with CMOS fabrication standards and low cost substrates with electronic performances consistent with the state of the art of AlGaN technology.

In this work we investigate in detail the feasibility of InAlN HEMTs as $\mathrm{pH}$ sensors, determining their surface sensitivity $\left(\Delta V_{\mathrm{sp}} / \Delta \mathrm{pH}\right)$ and current sensitivity $\left(\Delta I_{\mathrm{ds}} / \Delta \mathrm{pH}\right)$ working with an without reference electrode. As $\mathrm{GaN}$ has been recognized to have $\mathrm{pH}$-sensitivity of the order of the theoretical Nerstian limit $(59 \mathrm{mV} / \mathrm{pH}$ at room temperature) [6,7] we use HEMT structures always with a GaN capping layer as sensitive surface, but with different thicknesses of the AllnN barriers (either $6 \mathrm{~nm}$ or $10 \mathrm{~nm}$ ), so different distances between the surface and the 2DEG that suffers modulation due to surface potential changes.

\section{Experimental}

The samples used in this work are HEMT structures grown by MOCVD on silicon substrates, and their structure is schematized in Fig. 1a. An AlN nucleation layer of nominal $60 \mathrm{~nm}$ thickness was grown on high resistivity (HR) $\mathrm{Si}(111)$ substrate $(\rho>5 \mathrm{k} \Omega \mathrm{cm})$ and after that a HR GaN epilayer of $600 \mathrm{~nm}$. In a typical InAIN/GaN heterostructure, a very thin AlN interlayer (in this case, $1 \mathrm{~nm}$ thick) is introduced at the interface to improve electron confinement by increasing the effective barrier, and at the same time to reduce alloy scattering [15]. The InAlN barrier was of two different thicknesses, namely $6 \mathrm{~nm}$ (sample A) and $10 \mathrm{~nm}$ (sample B). Finally a GaN cap layer of $1 \mathrm{~nm}$ covered the samples.

$(0002) \theta / 2 \theta$ X-ray reflection diffraction (XRD) has been performed on the samples (not shown here). In the spectra, the GaN and AlN peaks are identified respectively at $\theta=17.283^{\circ}$ and $\theta=18.025^{\circ}$, as well as the band related to the InAIN barrier with a LM composition (at around $\theta=17.5^{\circ}$ for In content of $17 \%$ ).

We patterned the device structure with ICP dry etching (MESA) creating a channel between two ohmic contacts (Fig. 1b), having this conduction channel a length $(L)$ and width $(W)$ of $1.2 \mathrm{~mm}$ and $0.5 \mathrm{~mm}$, respectively. The ohmic metallization of source and drain contacts ( $S$ and $D$ in the image) consisted in a multilayer scheme of Ti/Al/Ni/Au $(30 \mathrm{~nm} / 200 \mathrm{~nm} / 50 \mathrm{~nm} / 150 \mathrm{~nm})$ annealed at $850^{\circ} \mathrm{C}$ during $30 \mathrm{~s}$, assuring a good contact resistance in all the samples investigated ( $R_{\mathrm{C}} \leq 1 \Omega \mathrm{mm}$ ). The resistance of the devices (in air) measured between source and drain was $1.5 \mathrm{k} \Omega$ (sample A) and $0.7 \mathrm{k} \Omega$ (sample B).

Previous to the characterization of the devices as $\mathrm{pH}$-sensor, we extracted the electron concentration in the 2DEG $\left(n_{s}\right)$ and the field effect mobility $\left(\mu_{\mathrm{fe}}\right)$ of the structures. By short circuiting the source and drain terminals and negative biasing the eletrolytegate with respect to them, we could extract the dependence of the density of electron from gate-source voltage $\left(n_{\mathrm{s}}\left(V_{\mathrm{gs}}\right)\right)$ by electrolyte capacitance-voltage measurements (ECV). The mobility is extracted from the source-drain current $\left(I_{\mathrm{ds}}\right)$ versus electrolytegate voltage $\left(V_{g s}\right)$ characteristics at a fixed low source-drain voltage $\left(V_{\mathrm{ds}}=50 \mathrm{mV}\right)$, together with the relation $n_{\mathrm{s}}\left(V_{\mathrm{gs}}\right)$ extracted before [16]. For sample $\mathrm{A}$, the maximum value of $\mu_{\mathrm{fe}}$ is $739 \mathrm{~cm}^{2} / \mathrm{V} \mathrm{s}$ when $n_{\mathrm{S}}=8.481 \times 10^{12} \mathrm{~cm}^{-2}$, and for sample B, $\mu_{\mathrm{fe}}=1283 \mathrm{~cm}^{2} / \mathrm{V} \mathrm{s}$ when $n_{\mathrm{S}}=6.955 \times 10^{12} \mathrm{~cm}^{-2}$. These values are consistent with the state of the art of AllnN/GaN HEMT structures grown on silicon (111) [17].

We treated the open gate of the devices with piranha solution $\left(1: 1 \mathrm{H}_{2} \mathrm{O}_{2}: \mathrm{H}_{2} \mathrm{SO}_{4}\right)$ to increase the native oxide $\left(\mathrm{Ga}_{x} \mathrm{O}_{y}\right)$ concentration, therefore the amphoteric hydroxyl groups responsible for $\mathrm{pH}$ sensitivity according to the site-binding theory [12]. In fact, we observed a clear increment in sensitivity after this treatment.

\section{Results and discussion}

The response to aqueous solutions with $\mathrm{pH}$ of 4,7 and 10 (commercial buffer solutions purchased at VWR) was systematically investigated at room temperature. Measurements of $I_{\mathrm{ds}}-V_{\mathrm{gs}}$ were recorded using an HP4156C parameter analyzer, while an HP4284 LCR-meter was used for capacitance-voltage measurements. An $\mathrm{Ag} / \mathrm{AgCl}$ electrode was used as the reference electrode during the measurements.

In Fig. 2 we report on the source-drain current $\left(I_{\mathrm{ds}}\right)$ and transconductance $\left(g_{\mathrm{m}}=d I_{\mathrm{ds}} / d V_{\mathrm{gs}}\right)$ versus $V_{\mathrm{gs}}$, at $V_{\mathrm{ds}}=200 \mathrm{mV}$ and $\mathrm{pH}=10$, for both samples $\mathrm{A}$ and $\mathrm{B}$. The maximum transconductance can be expressed as

$g_{\mathrm{m}}^{\max }=\left(\frac{d I_{\mathrm{ds}}}{d V_{\mathrm{gs}}}\right)^{\max }=\left(\frac{W}{L}\right) V_{\mathrm{ds}} \mu^{\max } C_{i}$,

where $C_{i}=\left(\varepsilon \cdot \varepsilon_{0}\right) / z$ is the capacitance per unit area between the electrolyte and the 2DEG channel, and $\mu^{\max }$ is the maximum electron mobility. Leaving post-growth design parameters $(W / L)$ and $V_{\mathrm{ds}}$ aside, $\mu / z$ is the main key parameter to obtain a high a

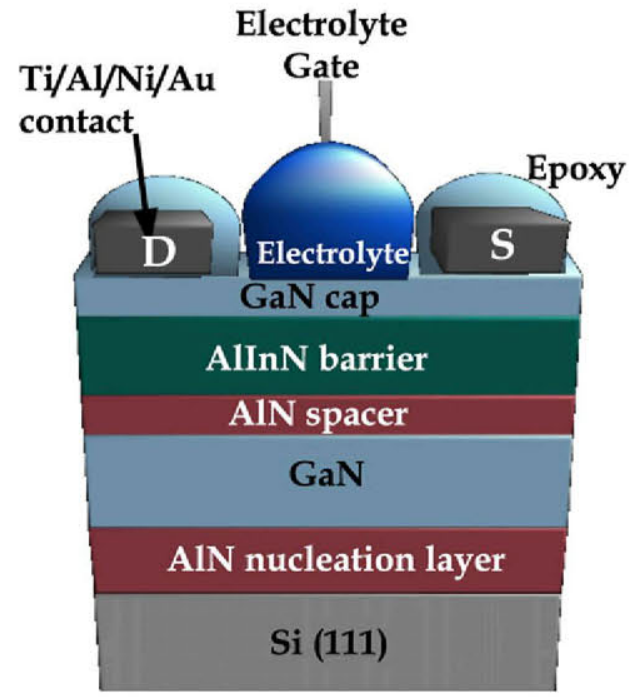

b

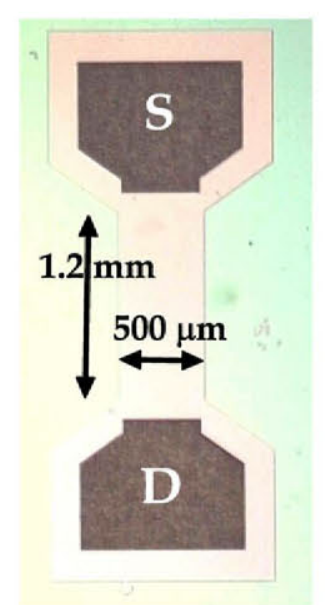

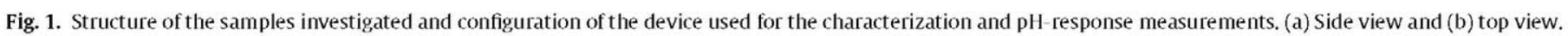




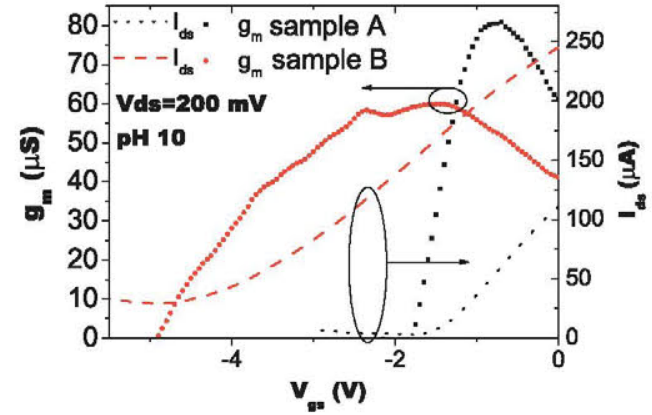

Fig. 2. Transconductance $\left(g_{\mathrm{m}}\right)$ and source-drain current $\left(I_{\mathrm{ds}}\right)$ versus gate voltage for the two samples recorded at $\mathrm{pH} 4$ and $V_{\mathrm{ds}}=200 \mathrm{mV}$.

transconductance value and therefore, high transduction sensitivity. The improvements in LM AllnN growth on GaN allow to create high quality AlInN/GaN HEMT structure with thin barrier (lower z) and keeping high mobility values.

The threshold voltage $\left(V_{\text {th }}\right)$ of the two devices rescale with the barrier thickness, being in the case of sample $A V_{\text {th }}=-1.8 \mathrm{~V}$ while in case of sample B $V_{\text {th }}=-4.9 \mathrm{~V}$. Moreover, higher maximum $g_{\mathrm{m}}$ is obtained for sample A which present a thinner barrier and a lower mobility in comparison with sample B.

We extracted the variation of the surface potential with the $\mathrm{pH}$ (Fig. 3) by fixing $V_{\mathrm{ds}}$ at $200 \mathrm{mV}$ and measuring $I_{\mathrm{ds}}-V_{\mathrm{gs}}$ for the three different $\mathrm{pH}$ values. Shifts on the $I_{\mathrm{ds}}-V_{\mathrm{gs}}$ curves depending on the $\mathrm{pH}$ reflect the surface potential dependence on the $\mathrm{pH}$ of the electrolyte, and the obtained slopes are $-53.7 \mathrm{mV} / \mathrm{pH}$ and $-52.1 \mathrm{mV} / \mathrm{pH}$ for samples A and B, respectively, close to the Nernstian theoretical limit at $25^{\circ} \mathrm{C}$ of $59 \mathrm{mV} / \mathrm{pH}$ [6].

Whereas the variation of surface potential depends on surface properties, the dependence of $I_{\mathrm{ds}}$ with the $\mathrm{pH}$ of the solution $\left(\Delta I_{\mathrm{ds}} / \Delta \mathrm{pH}\right)$ is strongly affected by the electrical characteristic of the structure and therefore by $g_{\mathrm{m}}$. In fact, the relationship between $\left(\Delta I_{\mathrm{ds}} / \Delta \mathrm{pH}\right)$ and $\left(\Delta V_{\mathrm{sp}} / \Delta \mathrm{pH}\right)$ is

$$
\left(\frac{\Delta I_{\mathrm{ds}}}{\Delta \mathrm{pH}}\right)=g_{\mathrm{m}}^{\prime} \cdot\left(\frac{\Delta V_{\mathrm{sp}}}{\Delta \mathrm{pH}}\right) \text {. }
$$

The results of $\left(\Delta I_{\mathrm{ds}} / \Delta \mathrm{pH}\right)$ for samples $\mathrm{A}$ and $\mathrm{B}$ are shown in Fig. 4. $V_{\mathrm{gs}}$ is set to the voltage at which the device shows its maximum $g_{\mathrm{m}}$, i.e. $V_{\mathrm{gs}}=-0.7 \mathrm{~V}$ for sample A, and $V_{\mathrm{gs}}=-2 \mathrm{~V}$ for sample B. In both cases $V_{\mathrm{ds}}=200 \mathrm{mV}$. A linear dependence has been observed in the two cases, with a higher slope for sample $A(4.16 \mu \mathrm{A} / \mathrm{pH})$ than for sample B $(2.83 \mu \mathrm{A} / \mathrm{pH})$, as expected due to the higher $g_{\mathrm{m}}$ of sample $A$ because of its smaller barrier thickness in spite of its lower mobility.

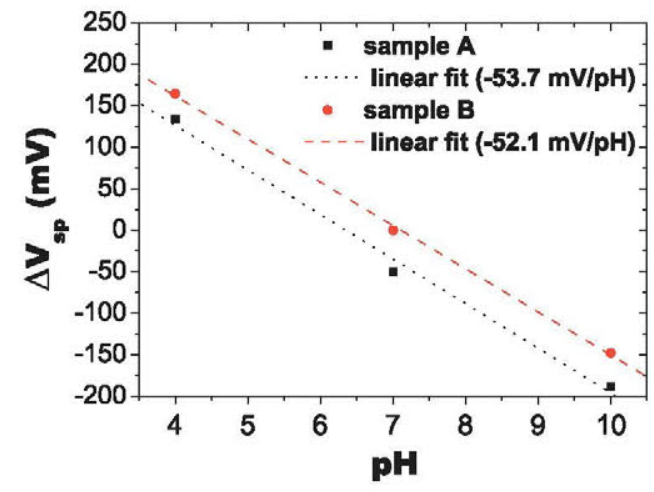

Fig. 3. Variation of the surface potential $\left(\Delta V_{\mathrm{gs}}\right)$ versus $\mathrm{pH}$ of the solution and linear fits for the two samples.

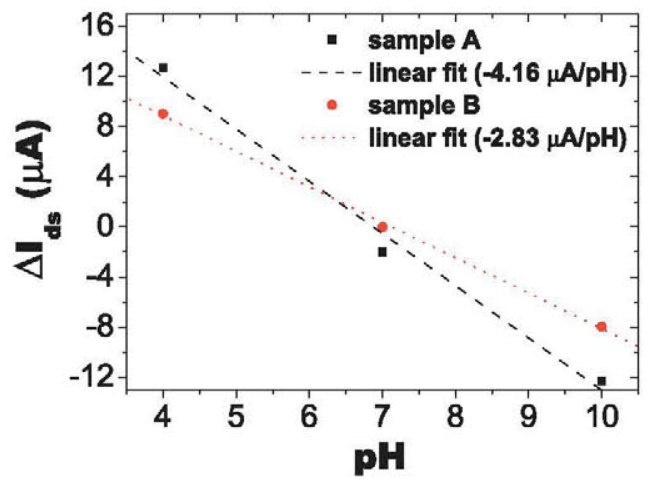

Fig. 4. Variation of the drain-source current $\left(\Delta I_{\mathrm{ds}}\right)$ versus $\mathrm{pH}$ of the solution and linear fits for the two samples.

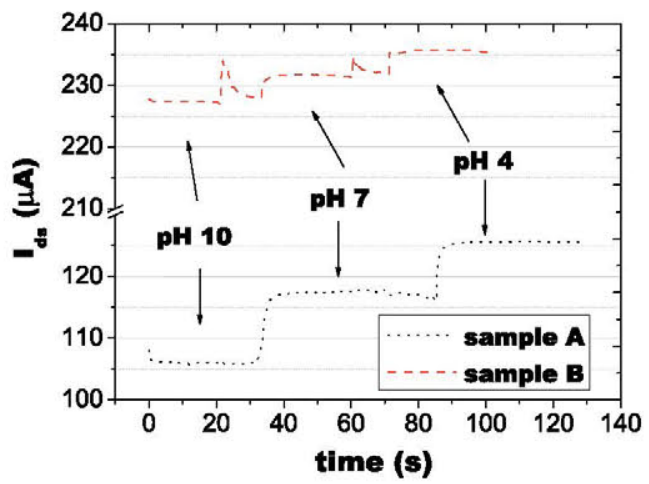

Fig. 5. Transient behaviors of the two samples when $\mathrm{pH}$ is changed by means of droplets of buffer solutions ( $\mathrm{pH} \mathrm{4,7}$ and 10), without reference electrode.

After that, we also performed real-time $I_{\mathrm{ds}}$ measurements for the two samples changing the $\mathrm{pH}$ of the solution without the use of an external reference electrode which bias at maximum $g_{\mathrm{m}}$. If possible, the current readout in $\mathrm{pH}$ sensing without the need of a reference electrode may be very practical. Without a reference electrode, the current $I_{\mathrm{ds}}$ is then just controlled by the surface potential that depends on the $\mathrm{pH}$ of the electrolyte, but most probably the operation point would be away from the maximum $g_{\mathrm{m}}$.

We fixed the drain-source voltage at $200 \mathrm{mV}$ as in previous experiment and measured the variation with time of the sourcedrain current by applying to the open gate a drop of the different buffer solutions by means of Pasteur pipettes (Fig. 5).

No substantial drift of the current was observed after the application of the buffer solution on the open gate, and high reproducibility and stability are observed.

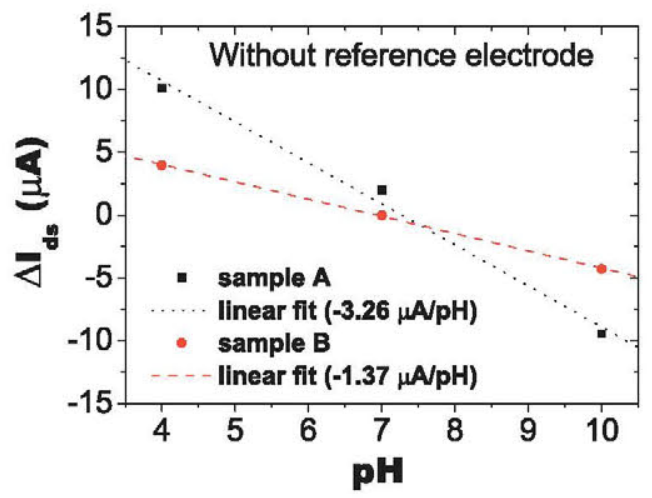

Fig. 6. Variation of the drain source current $\left(\Delta I_{\mathrm{ds}}\right)$ versus pH of the solution without the use of reference electrode, and linear fits for the two samples. 
A linear behavior of $\Delta I_{\mathrm{ds}}$ versus the $\mathrm{pH}$ of the solution is found (see Fig. 6), although as expected, working without reference electrode reduces the sensitivity of both devices. The response decrease in the sample A (from $4.16 \mu \mathrm{A} / \mathrm{pH}$ to $3.26 \mu \mathrm{A} / \mathrm{pH}$, reduction of $22 \%$ ) is less pronounced that in sample B (from $2.83 \mu \mathrm{A} / \mathrm{pH}$ to $1.37 \mu \mathrm{A} / \mathrm{pH}$, reduction of $52 \%$ ). This is reasonable taking into account that maximum transconductance for sample $\mathrm{A}$ is achieved at a $V_{\mathrm{gs}}$ value less negative (closer to zero) than in the case of sample B (see Fig. 2); thus, when working without reference electrode, sample $A$ is spontaneously closer to its maximum transconductance than sample B.

\section{Conclusions}

We have investigated the pH-sensitivity of InAlN/GaN heterostructure grown on Si substrates. The devices tested showed potential sensitivity of the order of the Nerstian limit. Even though the sample with larger barrier showed higher field effect mobility, the device with the thin barrier presented an enhanced $\Delta I_{\mathrm{ds}} / \Delta \mathrm{pH}$, related to the higher influence on the $2 \mathrm{DEG}$ density from the environment, being the 2DEG closer to the surface.

Measurements without reference electrode confirmed how the thin barrier improves the sensor performance working at a point closer to the maximum transconductance.

Therefore we can conclude that sample A, with a barrier thickness of only $6 \mathrm{~nm}$, has the best performance for $\mathrm{pH}$ sensing current readout; even working without reference electrode its sensing response is better than the one of sample B with a $10 \mathrm{~nm}$ barrier thickness.

In conclusion, the AlInN barrier ISFET with a LM thin barrier and grown on silicon is a very promising candidate for chemical sensors which can be integrated with CMOS technology.

\section{Acknowledgements}

This work has been performed in the EU frame FP7 of the "RAINBOW" Initial Training Network (PITN-GA-2008-213238), with partial support from Ministerio de Ciencia e Innovación of Spain by the RUE (CSD2009-00046) project.

\section{References}

[1] P. Bergveld, IEEE Transactions on Biomedical Engineering BME-17 (1970) $70-71$.

[2] J. Bausells, J. Carrabina, A. Errachid, A. Merlos, Sensors and Actuators B 57 (1999) $56-62$.

[3] P.A. Hammond, D.R.S. Cumming, Sensors and Actuators B 111-112 (2005) $254-258$.

[4] J. Schalwig, G. Müller, M. Eickhoff, O. Ambacher, M. Stutzmann, Materials Science \& Engineering B 93 (2002) 207-214.
[5] N. Chaniotakis, N. Sofikiti, Analytica Chimica Acta 615 (2008) 1.

[6] S.S. Kocha, M.W. Peterson, D.]. Arent, J.M. Redwing, M.A. Tischler, J.A. Turner Journal of the Electrochemical Society 142 (12) (1995) L238-L240.

[7] G. Steinhoff, M. Hermann, W.J. Schaff, L.F. Eastman, M. Stutzmann, M. Eickhoff, Applied Physics Letters 83 (2003) 177.

[8] Y.-H. Chang, Y.-S. Lu, Y.-L. Hong, S. Gwo, J.A. Yeh, IEEE Sensors Journal 11 (2011) $1157-1161$.

[9] A. Podolska, M. Kocan, A.M. Garces Cabezas, T.D. Wilson, G.A. UmanaMembreno, B.D. Nener, G. Parish, S. Keller, U.K. Mishra, Applied Physics Letters $97(2010) 012108$

[10] Y. Alifragis, A. Georgakilas, G. Konstantinidis, E. Iliopoulos, A. Kostopoulos, N.A. Chaniotakis, Applied Physics Letters 87 (2005) 253507.

[11] A. Bengoechea-Encabo, J. Howgate, M. Stutzmann, M. Eickhoff, M.A. SánchezGarcía, Sensors and Actuators B 142 (2009) 304307.

[12] W.M. Siu, R.S.C. Cobbold, IEEE Transactions on Electron Devices ED-26 (1979) 1805.

[13] F. Medjdoub, M. Alomari, J.-F. Carlin, M. Gonschorek, E. Feltin, M.A. Py, N. Grandjean, E. Kohn, IEEE Electron Device Letters 29 (2008) 422.

[14] H. Sun, A.R. Alt, H. Benedickter, C.R. Bolognesi, E. Feltin, J.-F. Carlin, M. Gonschorek, N. Grandjean, T. Maier, R. Quay, IEEE Electron Device Letters 30 (2009) $796-798$.

[15] R. Tülek, A. Ilgaz, S. Gökden, A. Teke, M.K. Öztürk, M. Kasap, S. Özçelik, E. Arslan, E. Özbay, Journal of Applied Physics 105 (2009) 013707.

[16] X.Z. Dang, P.M. Asbeck, E.T. Yu, G.J. Sullivan, M.Y. Chen, B.T. McDermott, K.S. Boutros, J.M. Redwing, Applied Physics Letters 74 (1999) 3890.

[17] N. Watanabe, H. Yokoyama, M. Hiroki, Y. Oda, T. Kobayashi, T. Yagi, Proc. of the Compound Semiconductor Integrated Circuit Symposium, 2006, pp. 257-260.

\section{Biographies}

T. Brazzini received his B.S. and M.S. in Physics from the Università degli studi di Firenze (2009) with a work on Er-doped silicon based light emitting devices. Currently he is working toward the Ph.D. degree at Instituto de Sistemas Optoelectronicos y Microtecnologia (ISOM-UPM) on indium containing nitrides characterization, device and sensor fabrication inside the RAINBOW-ITN European project.

A. Bengoechea Encabo received the B.E. and M.S. degrees in Physics from the Universidad de Valladolid (2002). After that she worked during 2 years on magnetooptics at Instituto de Microelectronica de Madrid (IMM-CSIC). In 2005 she moved to Instituto de Sistemas Optoelectronicos y Microtecnologia (ISOM-UPM), where she earned her Ph.D. on growth of nitrides heterostructures and nanocolumns and thei sensing applications.

M.A. Sánchez-García earned degrees in Electrical Engineering from Brown University (B.S. 1991, M.S. 1993) and from the Polytechnical University of Madrid (Ph.D. 2000). He has worked on MBE growth and characterization of III-V semiconductors at UPM since 1993. Since 2003 he is Associate Professor at the Department of Electrical Engineering at the School of Telecommunication (UPM). He carries out his current research at the Instituto de Sistemas Optoelectronicos y Microtecnologia (ISOM-UPM), focusing on the growth and characterization of III-nitrides layers and nanostructures for sensor applications and optoelectronic devices.

F. Calle received his Ph.D. in Physics at Universidad Autónoma de Madrid (Spain), he spent stays at the Max Planck Institute FKF (Germany) and Bell Labs (USA) From 1992 he is Professor in the Universidad Politécnica de Madrid, where he was Research Director of the Telecomm School, and Assistant Director of the Optoelectronics Systems and Microtechnology Institute. His research is related to the physics, technology, and applications of wide bandgap semiconductors and graphene (communications, power electronics, energy storage and harvesting, and harsh environments). He has been PI of 25 projects, and coauthored more than 200 international publications. 